Article

\title{
Self-Organised Approach to Designing Building Thermal Insulation
}

\author{
Purvesh Bharadwaj * and Ljubomir Jankovic \\ Zero Carbon Lab, School of Creative Arts, University of Hertfordshire, Hatfield, AL10 9AB, UK; \\ L.Jankovic@herts.ac.uk \\ * Correspondence: p.bharadwaj@herts.ac.uk
}

Received: 21 May 2020; Accepted: 15 July 2020; Published: 17 July 2020

\begin{abstract}
Traditionally, the uniform application of thermal insulation is practised within the built environment sector to achieve desired building regulation standards for energy efficiency. However, that approach does not follow the building heat loss field, and it is therefore poorly matched to the actual heat loss from the building, thus achieving sub-optimum energy performance. This research aims to visualise building heat loss field in three dimensions and to create self-organised thermal insulation patterns that are proportional in thickness to the intensity of heat loss. This is achieved using a 3D agent-based model, in which each agent that represents a miniature object of thermal insulation moves up the gradient of the heat loss representation and competes for its position with the neighbouring thermal insulation components, depending upon the gradient intensity. This creates a self-organised thermal insulation pattern through the competition between the thermal insulation components and through overcrowding in the areas with higher heat loss intensity. This helps to visualise the heat loss field and create a representation of thermal insulation that is ideally matched to it. The result is assessed for its energy performance using a conventional energy performance analysis. That analysis shows that this approach leads to reductions in energy consumption and carbon emissions in comparison with the conventional approach that uses the same amount of thermal insulation material. The overall result increases our understanding of 3D heat loss and introduces a new approach for designing building thermal insulation.
\end{abstract}

Keywords: thermal insulation agents; heat loss visualisation; emergence-based design; self-organised insulation; heat-loss-matched thermal insulation; emergent design solutions; emergent behaviour

\section{Introduction}

This research discusses the development of an emergent, self-organised approach to developing an energy efficient insulation design solution. Conventionally, buildings are insulated with a uniform thickness of insulation. But that approach does not follow the building heat loss field, and it is therefore poorly matched to the actual heat loss from the building, thus achieving sub-optimum energy performance. This research draws inspiration from natural processes such as the heat flow gradient to investigate the creation of 3D agent-based objects, by specifying behavioural rule sets for the interaction between insulation objects/building blocks and the environment. In this approach, each agent that represents a miniature object of thermal insulation moves up the gradient of the heat loss representation and competes for its position with the neighbouring thermal insulation components, depending upon the gradient intensity. This creates a self-organised thermal insulation pattern through the competition between the thermal insulation components and through overcrowding in the areas with higher heat loss intensity. This helps to visualise the heat loss field and create a representation of thermal insulation that is ideally matched to it. 


\subsection{Contemporary Building Simulation Methods and Their Shortcomings}

The conventional approach to building simulation and optimization, that leads to thermal insulation specification, stems from the developments in mathematical and scientific knowledge in the past few centuries. Methods such as the finite element method, topology optimization, shape optimization, etc., have gained significant popularity within the built environment sector, enabling professionals to examine their design in a new analytical plane. These methods gained recognition and wide acceptance in the structural field [1] and soon propagated into other fields, such as fluid flow [2], aeroelasticity [3], etc.

While these methods are able to generate solutions, they represent the problem domain in the form of a system of equations, which do not have complete solutions. As a result, the solutions developed are approximations. These methods provide a top-down approach where the problem is brought closer to the solution through simplification. Furthermore, complex solving methods such as the finite element analysis and Navier-Stokes equations require a significant number of iterations, information and assumptions to generate an output for a specified time period, and, in the case of the latter, cases that cannot be solved. This is in sharp contrast with how things work in nature, where heat loss and other phenomena occur through the interaction between the neighbouring components of the system, such as molecules, and through self-organisation of the system behaviour though the interaction of the constituent parts. Thus, the whole becomes more than the sum of parts.

Amongst the vast body of work exploring the application of component-based systems, Wolfram states that, despite the developments in traditional mathematics, it has only been able to reproduce the simplest of natural behaviours [4]. He further questions the holistic nature of the solutions developed through these methods. Arguing that, as contemporary building simulation tools utilize traditional mathematics that use systems of equations to explore and develop solutions, these solutions are incomplete and out of step with nature. Additionally, being based on incomplete formulations, these solutions result in a performance gap between the simulated and as-built results. These findings and arguments were further expanded in [5]. Due to the top-down nature of the approach towards modelling a physical system as a whole using systems of equations, a large number of iterations are required to achieve a solution. Additionally, the process also requires a large amount of information in great detail, resulting in a time-consuming model development process. Subsequently, as a result of the top-down approach of these methods, designers are forced to resort to a simplification of the problem to achieve solutions, while compromising the accuracy of results [5].

In order to overcome the shortcomings of the conventional approach, a more direct approach to simulation and investigation is required, such as self-organising emergent systems similar to those occurring in nature. As pointed out by Malina and Kauffman, the reductionist approach that is predominantly used to model natural processes presents limitations in explaining the complexity of nature [6].

In their 2019 paper, Bharadwaj and Jankovic suggest that natural phenomena, e.g., building thermal behaviour, are a result of component-based interactions devoid of complex equations [7]. The result of these interactions provided evidence of a much faster process than that presented by the top-down approach. Furthermore, Kauffman argues that the top-down approach is unable to explain complex natural behaviour [8]. He further states that it would require knowledge of initial conditions with infinite precision and great detail of computation, which is not possible to achieve. Instead, using simple behavioural rules on a component basis provides much more information on the emergent patterns and behaviour than that initially specified into the model. Thus, the emergent self-organised approach to simulation gives back more than what was explicitly programmed and enables a deeper understanding of natural behaviour.

The focus of this article is the use of agent-based models for the investigation of thermal insulation design solution in response to heat loss. The article will explain the implementation of simple behavioural rules applied to different components. These rules will make each agent that represents a miniature object of thermal insulation move up the gradient of the heat loss representation and 
compete for its position with the neighbouring thermal insulation components. As result of such competition, and through overcrowding in the areas with higher heat loss intensity, a self-organised thermal insulation pattern will emerge. This will help to visualise the heat loss field and to create a representation of thermal insulation that is ideally matched to it.

The result will be assessed for its energy performance using a conventional energy performance analysis and compared with the conventional approach that uses the same amount of thermal insulation material.

\subsection{Previous Work}

There is a notable body of work focusing on the use of component-based models such as Cellular Automata (CA) to study various natural behaviours. One of the most notable works is that of Hardy, Pomeau and dePazzis, whose research focused on the recreation of fluid behaviour on a square lattice that allowed orthogonal interactions between fluid particles [9]. The resulting model from this research is known as the HPP model, named after the initial letters of the authors' surnames. This model was superseded by the work of Frisch, Hasslacher and Pomeau, which illustrated how diagonal interactions in six directions were possible using a hexagonal grid [10]. Building upon this work, Salem and Wolfram illustrated the development of an emergent model of hydrodynamic flow around a plate [11]. Subsequently, Wolfram published a theory on CA fluid [12]. Toffoli and Margolus also worked on CA-based emergent models simulating flow tracing, flow around obstacles circular waves, etc. [13]. They advocated the use of CA as a means of delivering solutions, as it is devoid of numerical instabilities, guided by the notion that "when things are what they seem, we can safely let them do what they must" [13]. Furthermore, CA models have been increasingly used in a variety of disciplines, such as social sciences, medical sciences, urban traffic and public movement, etc.

There exists very little literature to show the use of component-based models including CA within the built environment sector. Frazer investigated the fundamental form-generation processes in architecture, with the aim of developing models with "nature as the generating force for architectural forms" [14]. Through various models governed by the application of either simple behavioural rules or genetic algorithms, that research illustrated how a symbiotic behaviour between the built environment and nature can be achieved, enabling explorations of architectural design forms. Jankovic and co-workers investigated an emergence-based self-organised approach using independent component objects. This work illustrated how a dynamic and more realistic behaviour of bridges could be reproduced, in comparison with the top-down approach based on the finite element method $[15,16]$.

A further development of this work by Jankovic illustrated how emergence-based models can be utilized to produce emergent behaviour and self-organising patterns of building forms using attractors, representing circulation areas within the model space [17]. The resulting emergent patterns from these experiments demonstrated how building typologies and building forms can be explored, also resembling some well-known building forms. The results of a further development using a 2D CA approach by Jankovic achieved interactive fluid flow behaviour, enabling sketch models to quickly and interactively explore air flow patterns [5]. These models were based on Fourier's law of heat transfer and simple component-based interaction rule sets. The resultant interactive representation of air movement in building enclosures was achieved without solving complex Navier-Stokes equations, whilst implementing Newtonian physics on a component level.

The use of 2D CA models to investigate insulation design in response to heat loss through the building fabric was also explored by Bharadwaj and co-workers [18]. The 2D models were based on Fourier's law of heat transfer and simple rule sets for component-to-component interaction. The models developed in that research visualised the complex heat loss process through the building fabric, identifying areas of the building geometry that are significantly affected by the heat loss. Additionally, the models also illustrated the emergent behaviour and self-organising insulation pattern which developed as a result of the component-based interactions, in response to the heat loss process. The self-organised patterns from these models were analysed for their effectiveness using conventional 
building energy performance simulation tools. The results from these analyses illustrated that the energy performance of the emergent patterns was better in comparison with the conventionally designed solutions.

This research was further developed by the same team to simulate the convection behaviour of air objects within the model space and to investigate the self-organisation of insulation objects when externally instantiated to the building [7]. The self-emergent patterns from this research illustrated areas with a high degree of heat loss. Furthermore, a comparative analysis of these self-organised patterns illustrated a higher thermal performance by up to $10 \%$ in comparison to conventionally designed solutions.

\section{Methods}

The method in this article consists of three parts, and it stems from the research undertaken by Bharadwaj and co-workers [7,18]. In their publications, the authors argued that the 2D CA model comprises three stages:

1. Development of a CA model simulating the natural phenomenon of heat loss through the building fabric.

2. Capture and assessment of the self-organised emergent pattern using conventional tools such as those used in [19].

3. Development and refinement of a solution based on the emergent behaviour of the model.

The key findings of this research were:

1. The model that was developed indicated that the resultant self-organised solutions closely matched the complex processes found in nature.

2. The analysis of the generated self-organised solutions in response to the complex process of heat loss through the building fabric showed a better thermal performance than that of the solutions developed using the conventional design approach.

3. The developed CA models facilitated the visualisation of the complex heat loss pattern, thus enabling the identification of areas of the building fabric significantly affected by the heat loss.

4. The models that were developed helped to increase the understanding of the building behaviour.

The method employed in this research is similar to the one mentioned above. However, there are some key differences:

1. The models developed in this research are based on 3D objects of thermal insulation.

2. Heat transfer in these models has been replaced with a model of Newton's law of gravitation with reversed sign to investigate an alternative approach to modelling heat transfer.

3. Insulation cells are instantiated internally to the building to investigate the generation of a 3D self-emergent pattern of insulation cells.

4. The emergent self-organised pattern of insulation objects is tested for energy performance using a conventional simulation tool and compared with the conventional approach to insulation application that uses the same amount of thermal insulation material.

5. If the energy performance comparison between the case with emergent insulation pattern and the case with conventional insulation that uses the same amount of insulation material is favourable in terms of the former, that will justify the approach using Newton's law of gravitation with reversed sign to generate the emergent insulation pattern.

\subsection{Repellent-Based Approach}

At the start of this research, a 3D CA model was developed based on the methodology previously developed by the authors [7]. That model was developed applying Fourier's law for heat transfer on a 
component basis. However, a significant reduction in simulation performance was observed during runtime. This behaviour was attributed to the large number of objects instantiated within the simulation environment outside of the building enclosure. As each cell performed its calculations, ascertaining the cell temperature and movement along the heat loss gradient, several hundred processing threads were created. Hence, it was decided to investigate an alternative approach using evidence from previous research undertaken by Jankovic where he developed 3D models based on the principles of emergence, explaining that "emergence has been at the heart of nature's designs, producing complex living organisms for several millennia" [17]. In these models, attractors were placed in different locations of the simulation environment while building objects were randomly instantiated. The attractors dissipated "attractiveness" in the 3D space, so that a gradual reduction of the attractiveness intensity occurred, proportional to the square of the distance from the attractor, and the building objects followed the increasing gradient of attractiveness towards the attractors during runtime. This led to a self-organisation of the building objects, resulting in a building form and building typology.

Thus, it was decided to explore the use of similar concepts for the creation of this 3D model; however, instead of using attractors, the models in this research utilized the concept of "repellents", based on Newton's law of gravity with a negative sign.

What is the rationale for using the repellents? According to Fourier's law, heat loss is proportional to the negative gradient in temperature and area, i.e., heat flow through a material occurs from a higher to a lower temperature. A repelling force is also an outwardly facing force, mathematically similar to that of heat loss, resulting in an outwardly movement of objects. Hence, it was decided to investigate if this concept can be utilized to simulate the representation of heat loss using Newton's law of gravity with inverse sign.

If the emergent self-organised pattern of thermal insulation representation resulting from this approach appears to be better in terms of energy performance in comparison with a conventional thermal insulation using the same amount of insulation material, that would be a justification for using the repellent force to generate the insulation pattern. The model development is introduced in the next section.

\subsection{Model Development}

This section will discuss the development of the 3D models and the implementation of the concept of repellents. The models that were developed share some common attributes with those developed by the authors $[7,18]$ in that they comprise two major components:

- A global environment;

- Cells/building blocks.

Bharadwaj and co-workers explain the role of these components as follows: a global environment provides the cells/building blocks with a traversable space, thus enabling their interaction with other components and with the environment [18]. In this research the global environment is provided by the Unity Game Development Platform [20], chosen for its physics engine capability and the 3D vector calculus. The building blocks in this simulation are analogous representations of the different building components and comprise different object types as follows:

- $\quad$ Repellents, representing the heat source;

- Brick, representing a building envelope component;

- Insulation, representing the insulation agent for the creation of the self-organised insulation patterns.

Subsequently, the model measuring $3 \mathrm{~m} \times 3 \mathrm{~m} \times 3 \mathrm{~m}$, with transparent walls, was placed within the unity environment, as illustrated in Figure 1a. The boundary conditions of the simulation environment are specified by the transparent walls which mark the physical limits of the simulation test space within the Unity Game Development Platform [20]. The movement of the cells was limited to the simulation test space. To simulate a uniformly heated internal environment within the room, a repellent object was 
placed in the centre of the room (Figure 1b), thus, specifying the initial conditions for the simulation. After these conditions were specified, the insulation objects were instantiated inside the room.

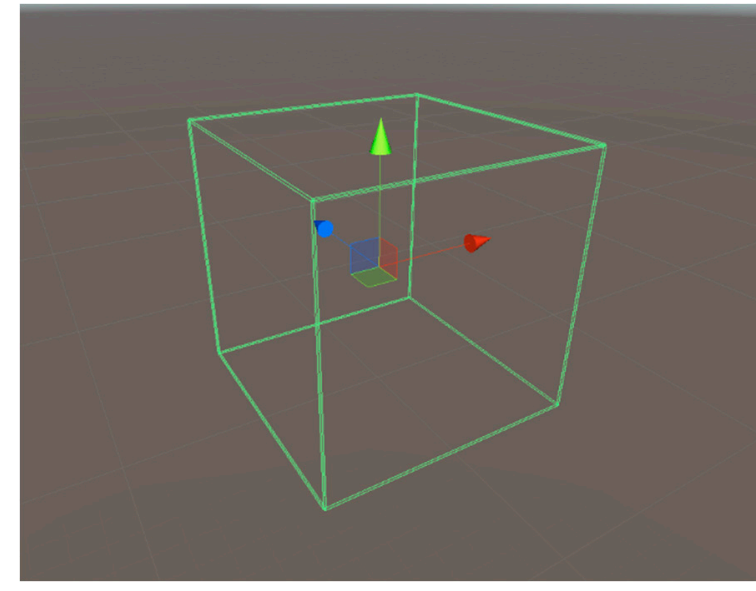

(a)

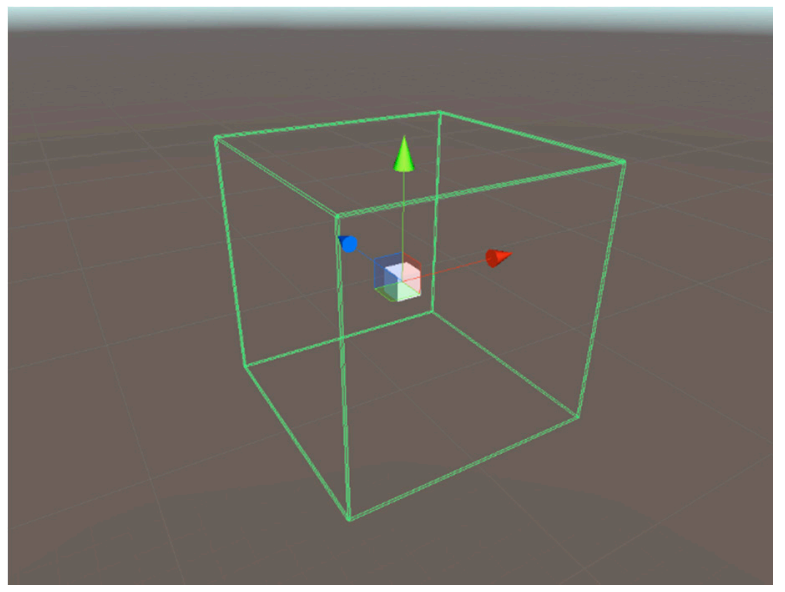

(b)

Figure 1. Setting up the simulation environment: (a) 3D room with transparent walls representing the simulation environment; (b) Placement of the repellent object within the room.

Model Physics

The overall model physics is governed by the application of Newton's law of gravity, with the exception that the gravitational attraction force between cells was replaced with the repulsion force by reversing the force sign, as explained below, in Equation (1):

$$
F_{\text {net }}=-G \cdot M_{1} \cdot M_{2} / d^{2}
$$

where:

$\mathrm{F}_{\text {net }}$-net force experienced by a cell $\left(\mathrm{kg} \cdot \mathrm{m} \cdot \mathrm{s}^{-2}\right)$

G-gravitational constant $\left(\mathrm{m}^{3} \cdot \mathrm{kg}^{-1} \cdot \mathrm{s}^{-2}\right)$

$\mathrm{M}_{1}$-mass of the repellent $(\mathrm{kg})$

$\mathrm{M}_{2}$ - mass of the cell (kg)

$\mathrm{d}$-distance between the cell and the repellent $(\mathrm{m})$.

The repulsive strength of the repellent in the model is specified by instantiating it with a high mass, in comparison to other object types in the model. The speed and direction of the movement of the objects within the simulation environment was calculated by the Unity Game Development Platform [20], as a response to the repulsive force experienced by each insulation component.

\section{Simulation Experiment}

A box model simulation experiment was undertaken to investigate the appropriateness and effectiveness of the repellent-based approach. Once the initial conditions for the model were specified, insulation objects were instantiated inside the room during runtime, as illustrated in Figure 2a. These objects were instantiated at a "key stroke" command, launching 100 insulation components with each key stroke in random positions inside the room (Figure 2b). Almost immediately upon the instantiation of the cells, a self-organised behaviour emerged (Figure 3a), as the insulation objects were driven outwards by the repellent. As can be seen in Figure 3b, with the further instantiation of the insulation cells or objects, an emerging pattern begins to form, as the objects compete to move into positions with the lowest intensity of repellent force. Due to this competition, an overcrowding occurs in the areas with the lowest repellent intensity. Finally, as the process continues, a stable self-organised pattern emerges, as illustrated in Figure 4. 


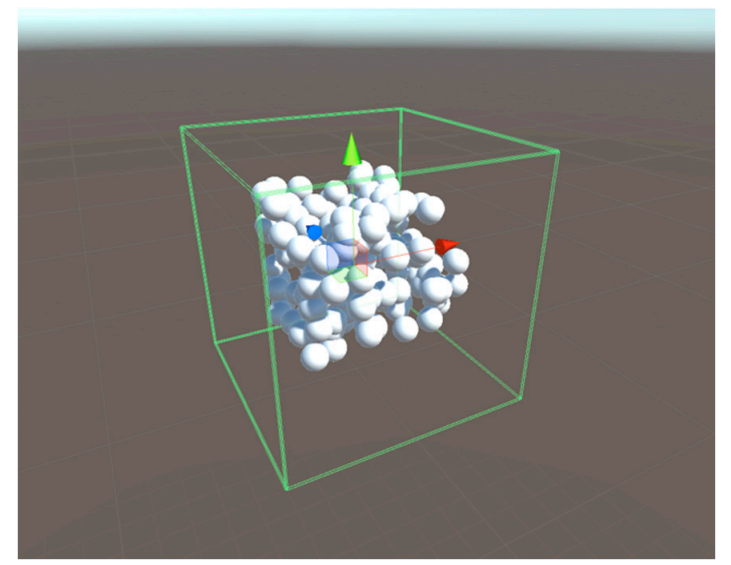

(a)

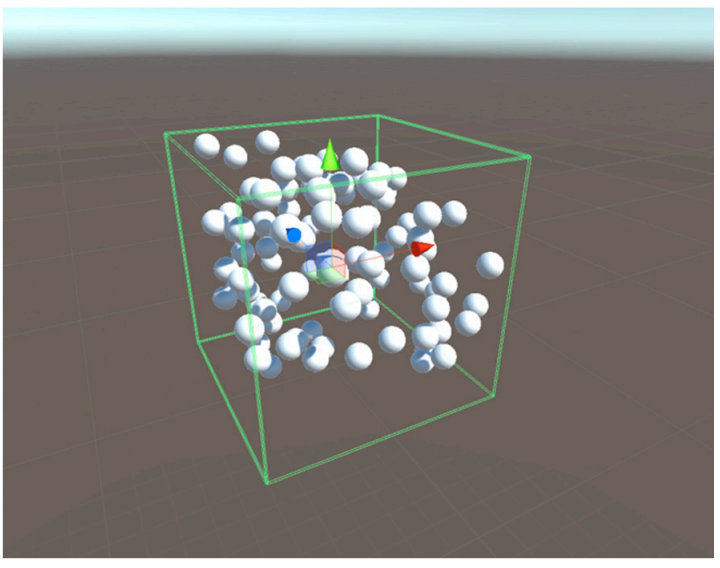

(b)

Figure 2. (a) Insulation objects are instantiated within the room 100 at a time; (b) Movement of insulation objects as they experience repelling forces.

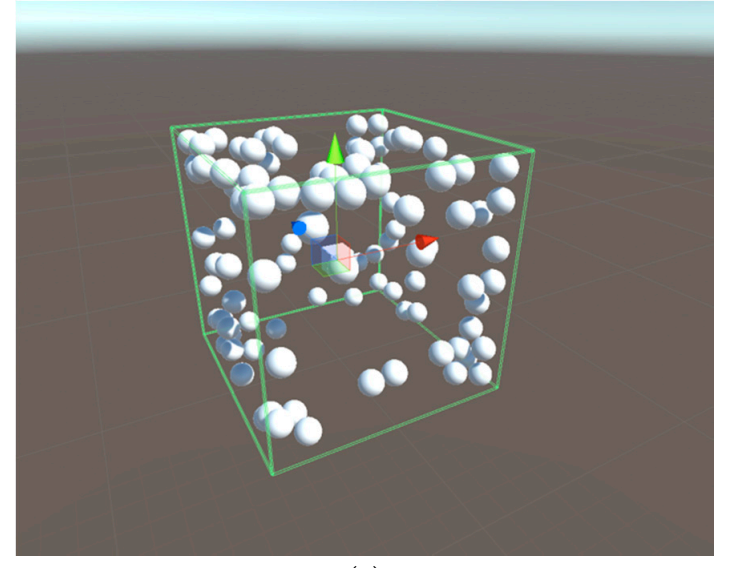

(a)

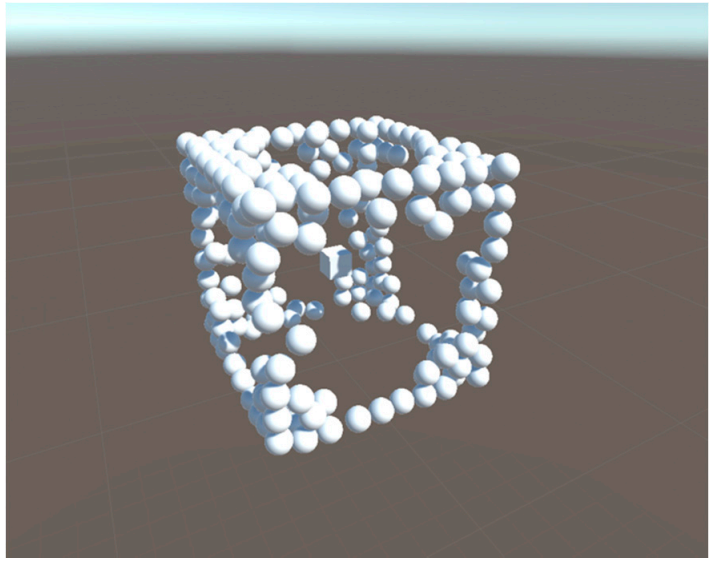

(b)

Figure 3. Formation of an emergent pattern: (a) Initial movement and formation of a self-organised behaviour; (b) Emergent pattern exhibited by the insulation objects.

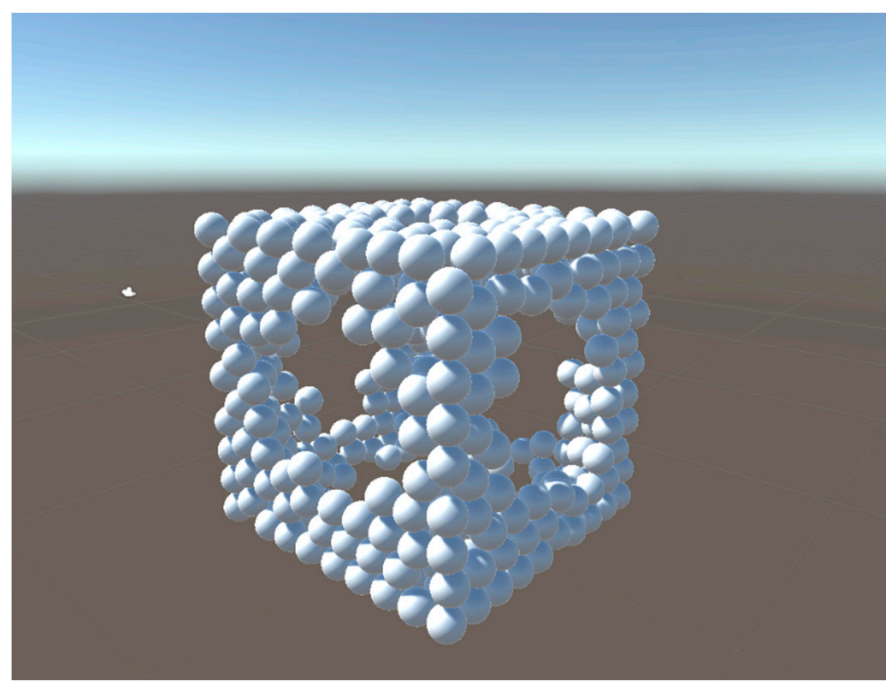

Figure 4. Creation of a stable self-organisational pattern of insulation. 
The simulation process illustrated from Figure 1a to Figure 4 was completed in less than a minute, and the process continued until manually terminated.

\section{Comparative Simulation Analysis}

Once a stable self-organised pattern was observed in the simulation model, it was possible to analyse its energy performance. In their paper on self-organised insulation patterns in 2D [7], the authors investigate and discuss the development of a comparative simulation analysis approach. That approach consisted in developing two models with identical building geometry $(3 \mathrm{~m} \times 3 \mathrm{~m} \times 3 \mathrm{~m})$ and with no windows and doors in a conventional building performance simulation tool [19]. The first model was a representation of the emergent insulation pattern obtained from the self-organised simulation. The second model was made with the conventional insulation approach of straight insulation panels, and with the same amount of insulation material as the first model. Furthermore, the building construction specified in both models was devoid of high-density materials such as concrete, brick, etc., and was based on thermal insulation only. Internal heat gains from lighting, people and appliances were set to zero. The model heating system was specified with a natural gas boiler with $89 \%$ efficiency, and with a heating set-point of $21^{\circ} \mathrm{C}$. A weather data file for Birmingham, UK, was specified for both models, as obtained from [19]. The computation period for the simulation was set to run for one year on an hourly timestep. These specifications formed the initial input conditions for the comparative simulation analysis. The results obtained from this approach illustrated the improved energy efficiency of the insulation design based on the self-organised emergent pattern. Thus, it was decided to investigate the performance of the $3 \mathrm{D}$ emergent pattern in this research using the same approach.

Two iterations of the model were developed. In the first iteration, the model was tested using the emergent pattern obtained from the 3D simulation, and in the second iteration the model was tested using a uniform insulation pattern obtained using the same amount of insulation material as in the first iteration.

There are several ways in which the absolute values of insulation in the corners of the emergent pattern in Figure 4 can be interpreted for transferring the insulation thickness to a conventional analysis tool. However, attempting to use absolute values based on the sizes of insulation objects may lead to unrealistic insulation thicknesses because of the relative scales chosen in the simulation. For that reason, relative insulation thicknesses were used. Hence, it appears that the corner insulation thickness in Figure 4 is generally two times higher than in the other insulated parts. For that reason, the insulation thickness adopted for the corners of the first iteration model (Figure 5a) was $400 \mathrm{~mm}$, extending $900 \mathrm{~mm}$ in each direction from the corner, and for the remaining surfaces the insulation thickness adopted was $200 \mathrm{~mm}$.

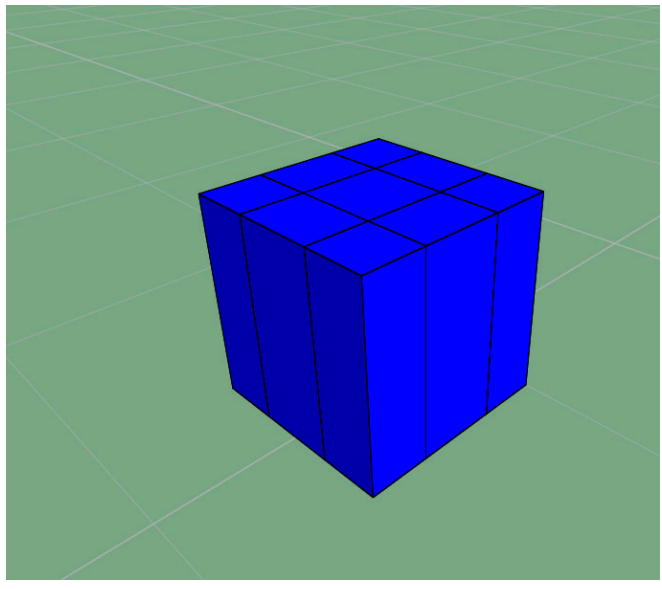

(a)

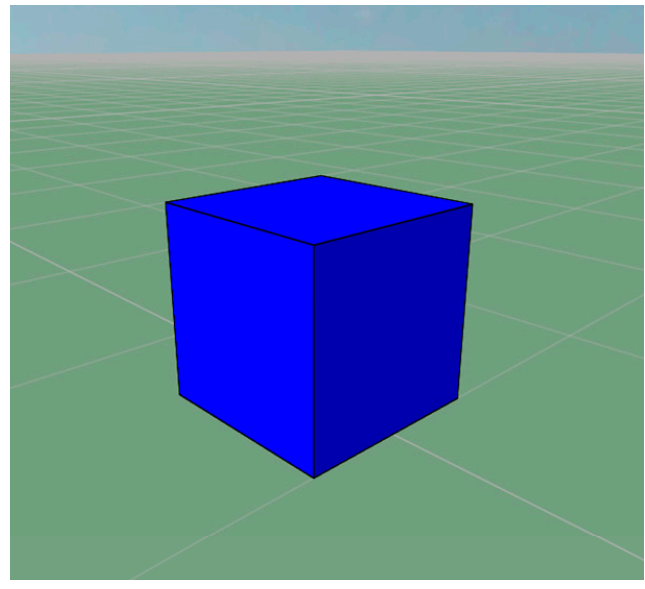

(b)

Figure 5. Box model iterations for comparison in the conventional simulation tool IES Virtual Environment [19]: (a) Emergent pattern box model representation; (b) Base case uniform pattern box model representation. 
In the base case model (Figure 5b), the insulation thickness was calculated by dividing the volume of insulation used in the emergent pattern case by the total surface area of the box, so that the same amount of insulation material was used in both cases. The resultant uniform insulation thickness was calculated to be $304 \mathrm{~mm}$.

As IES Virtual Environment [19] does not appear to take into account the thermal bridge, other alternatives were investigated. DesignBuilder [21] was one of these alternatives, as it enables linear thermal bridges to be specified. However, these specifications could not take into account different thermal insulation thicknesses. A third alternative investigated for taking into account thermal bridges was AnTherm [22]. It was found that this tool facilitates the calculation of linear thermal bridges depending on the values of overall thermal transmittance of the building components in direct contact, such as wall corners, wall-to-floor and wall-to-ceiling edges. That calculation facilitated the adjustments of the insulation thicknesses to enable a dynamic simulation to be carried out in IES Virtual Environment [19].

The overall heat loss coefficients for the emergent pattern and uniform pattern, adjusted for heat loss from the thermal bridges, is shown in Table 1. As can be seen from this table, the heat loss in the emergent pattern box model was reduced by $8.7 \%$ in comparison with the uniform pattern box model.

Table 1. The overall heat loss coefficients for the emergent pattern and uniform pattern adjusted for heat loss from thermal bridges.

\begin{tabular}{ccc}
\hline Model Iteration & $\begin{array}{c}\text { Overall Heat Loss Coefficient from } \\
\text { Conduction and Thermal Bridging (W/K) }\end{array}$ & $\begin{array}{c}\text { Heat Loss Coefficient } \\
\text { Reduction }\end{array}$ \\
\hline Emergent Pattern Box Model & 341.0 & $8.7 \%$ \\
Uniform Pattern Box Model & 374.3 & \\
\hline
\end{tabular}

The total energy consumption from the simulations of the two boxes from Figure 5, illustrated in Table 2, shows an improved performance of the emergent self-organised insulation. As can be seen from this table, the energy consumption reduction was 3.6\%, therefore lower than the heat loss reduction in Table 1. We believe this is because the thermal bridge heat losses were calculated for a static temperature difference between $20^{\circ} \mathrm{C}$ internal temperature and $-5^{\circ} \mathrm{C}$ external temperature by the AnTherm software [22], whilst the dynamic simulation used all hourly temperature differences between the calculated internal temperature and the external air temperature from the weather data file. Additionally, the two boxes have only been simulated for heating, and therefore not all of the heat loss reduction coefficient reduction has been reflected in the energy consumption reduction. The carbon emissions, being proportional to energy consumption, were also reduced by $3.6 \%$.

Table 2. Simulation results for box model iterations using IES Virtual Environment [19].

\begin{tabular}{ccc}
\hline Model Iteration & $\begin{array}{c}\text { Total Energy Consumption } \\
\text { (MWh) }\end{array}$ & $\begin{array}{c}\text { Energy Consumption } \\
\text { Reduction }\end{array}$ \\
\hline Emergent Pattern Box Model & 230.0 & $3.6 \%$ \\
Uniform Pattern Box Model & 238.6 & \\
\hline
\end{tabular}

Simulations of different geometries, different scales and different numbers of insulation objects will inevitably result in different performance improvements. However, the performance improvement of the emergent self-organised insulation has justified the use of the repellent force in this approach.

\section{Discussion and Analysis}

The simulation experiment explained in this article has illustrated the creation of a self-organised emergent thermal insulation pattern based on the implementation of Newton's law of gravity with a reversed sign. The justification of this approach is explained in the text below. 
At the start of this research, an initial 3D model created on the basis of Fourier's law exhibited emergent self-organised behaviour, but only a small number of insulation objects could be used due to the slow simulation performance. The component-to-component interaction in that initial model was based on a 3D "Moore neighbourhood" concept where each cell communicated with 27 other cells in the immediate neighbourhood, to calculate their temperature and movement along the heat gradient. The insulation objects were instantiated outside the building box enclosure, and that required a large number of objects to achieve self-organised behaviour. In a model with approximately 1000 insulation objects, this process resulted in 27,000 processing threads, impacting on the speed of simulation. Due to the slow simulation time attributed to this approach and to the requirement of instantiating a large number of insulation objects outside of the building box enclosure, emergent self-organised insulation patterns could not be effectively created.

For that reason, an alternative approach with a repellent gravitational force based on Newton's law of gravity with a reversed sign was investigated. This approach made it possible to instantiate a smaller number of insulation objects inside the building box enclosure. The objective was to find out if the resultant emergent self-organised pattern of thermal insulation representation would be better in terms of energy performance in comparison with a conventional thermal insulation using the same amount of insulation material. As can be seen from Table 1, this performance improvement has been achieved, thus justifying the repellent gravitational force approach.

Both approaches investigated in this research, Fourier's law of heat transfer and Newton's law of gravity, are based on the use of a simple and single equation which mathematically describes the complex natural behaviour of heat transfer and gravitation. In comparison, conventional approaches such as the finite element method involve complex systems of equations which produce solutions that require approximations and a large number of iterations, making it a resource intensive and a time-consuming process. In the case of the methods introduced in this article, the complex systems of equations are replaced by the interaction of the insulation objects with the repellent object, and the interaction of the insulation objects with each other.

The results from the above simulation experiment illustrate the creation of a self-organised emergent pattern of thermal insulation, in response to the repellent force used as a representation of heat loss. At the start of the simulation experiment, it was expected that the insulation objects would take some time to exhibit emergent behaviour; however, that behaviour occurred almost instantaneously, as the insulation objects were instantiated. The 3D patterns generated in the simulation help to visualise the heat loss field and create a representation of thermal insulation that is ideally matched to it. This process highlights the areas of the building that are more affected by heat loss, such as wall-to-wall, wall-to-ground and wall-to-roof junctions. The emergent pattern in Figure 6 is consistent with the 2D emergent pattern generated by the simulation experiments developed by the authors $[7,18]$, as shown in Figure $7 \mathrm{a}, \mathrm{b}$. In this research, the 2D emergent patterns exhibited a similar behaviour in response to heat loss, highlighting areas that are significantly affected by heat loss. The insulation object in the present experiments also exhibited an emergent behaviour of overcrowding, as each object competed with other objects in the simulation for the position furthest from the repellent under the constrained 3D space. This competing behaviour was a result of self-organisation in response to each object implementing Newton's law of gravity with a reversed sign, whilst the system's behaviour was not explicitly programmed and emerged through self-organisation.

While the 3D and 2D proofs of the concept models shown in Figures 6 and 7, respectively, do not claim to represent and reproduce natural behaviour accurately, they do illustrate a strikingly similar behaviour. Thus, these emergent patterns can be coupled with the ideas developed by Toffoli and Margolus, who argue that "when things are what they seem, we can safely let them do what they must" [13], and be utilized as a starting point for design exploration. Since the 3D model developed in this research only provides qualitative results of insulation design, it is important for these to be quantified and assessed for practical use. This can be achieved using conventional tools such as IES Virtual 
Environment [19], as demonstrated in the comparative simulation analysis section of this paper and summarized in Table 1.

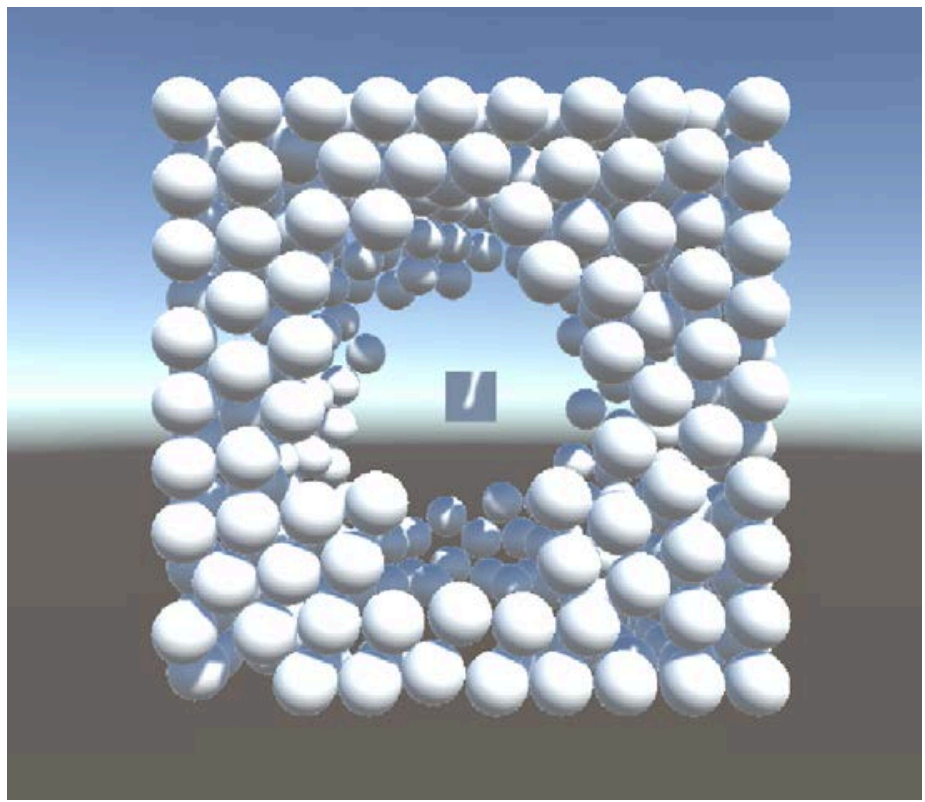

Figure 6. Isometric view of the emergent pattern box model.

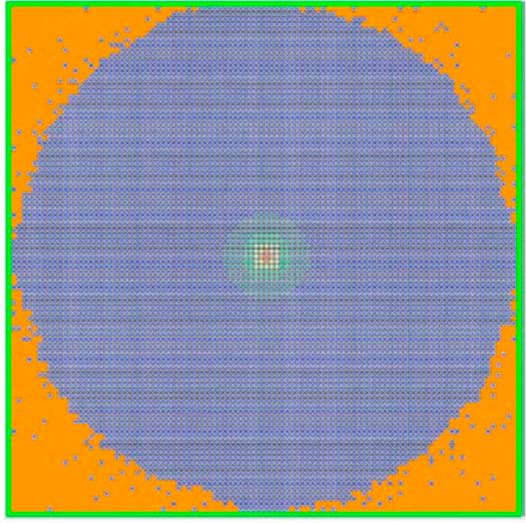

(a)

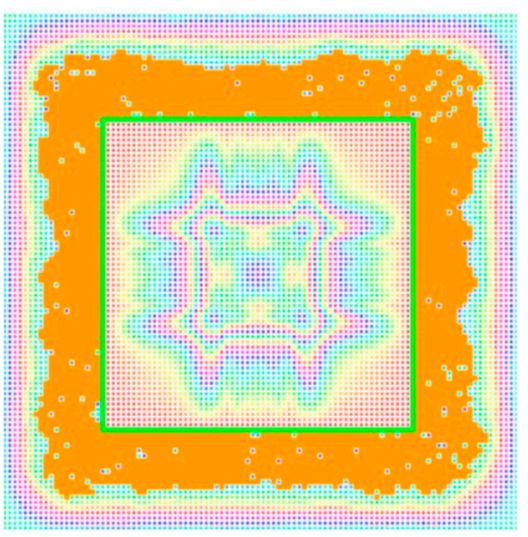

(b)

Figure 7. 2D Cellular Automata models: (a) 2D plan view model with internally instantiated insulation cells; (b) 2D plan view model with externally instantiated insulation cells, visualising the heat loss process.

Can it be said that the only thing this method is able to do is to identify geometric thermal bridges already well known by designers? We believe that this work goes well beyond that. It creates a 3D visualisation of the heat loss field and a representation of thermal insulation that is ideally matched to the heat loss field. Although designers generally know about thermal bridges, they do not have an insight into what the 3D heat loss field looks like, and this article introduces that insight.

Is the lack of differentiation between the different thermal elements of the building a shortcoming of this model, considering that the insulation thickness on the roof should be higher than that of the external walls, which should be higher than that of the ground floor? Whilst the authors acknowledge that this differentiation exists in practical design applications, it was outside the current scope of this research and will be added to our future versions of the model by changing the boundary conditions. However, our point is that the emergent self-organised approach introduced in this article offers new 
insights into the visualisation of the 3D heat loss field and the ideal matching of thermal insulation representation to the heat loss field. This has not been done before in a 3D domain, as building designers have not been trained to think along these lines.

Designers do not propose organic shapes as design solutions to alleviate thermal bridging. The self-organisation approach to insulation design presented in this research, though in its infancy, is able to visualise and highlight areas significantly affected by heat loss through the building fabric and does not require complex systems of equations. The visualisation of heat loss through the accumulation of insulation objects provides designers with a new plane of design exploration and allows for a stronger understanding of building physics. The model developed in this research is dynamic and instantly responsive to changes made during runtime. In comparison, conventionally developed simulations are only able to provide static time shot results for building performance.

The above suggests the following question: Can this work be regarded as being in a such an embryonic state that it is not worth publishing about? The point we are making is that, although some 6000 years have passed since the first differentiation between the endeavours of human hunter-gatherers and individual disciplines, that occurred at the onset of the agricultural revolution [23], building design has always been an essentially top-down activity, producing forms that do not accurately relate to the heat transfer processes they contain. This research re-addresses that ongoing disparity between nature and the representation of nature, and we believe that it is worthwhile publishing its results.

As real buildings are much more complex than the model presented in this article, should we have waited until more complex examples of the application of this work were developed? We believe that the changes in building design that this article advocates are so fundamental that we do not require more complex examples to demonstrate the concept, and that the simplest of examples, as presented here, is the best way to make our point. However, we will proceed to investigate more complex buildings and to vary the initial and boundary conditions.

How can the approach introduced in this article help designers? The model developed in this research was specified with a minimum amount of information, based on its initial conditions, and yet it was able to provide much more information than it was supplied with. Therefore, this creates opportunities for designers to analyse their design strategies quickly and from a very early stage of the design process. The dynamic nature of this simulation will provide designers with a stronger understanding of a building's thermal behaviour, enabling them to explore a vast design space. This is an improvement with regard to the static process, which requires vast amount of information and lengthy computing periods.

\section{Conclusions}

This article has introduced the development of a 3D self-organised simulation model based on the use of repellents to investigate whether it can re-create the complex natural phenomenon of heat loss through the building fabric. It has resulted in the visualisation of the heat loss field and the representation of the thermal insulation pattern that is ideally matched to that field.

A comparison between the generated emergent pattern and a conventional pattern using the same amount of thermal insulation material showed lower energy consumption and associated carbon emissions.

As this approach provides much more information to the user than the one initially supplied to the model, it presents opportunities for designers to investigate various design strategies at an early phase of the design process. Additionally, as the approach visualises the generation of the self-organised emergent insulation patterns, it provides a much greater insight into the complex natural heat loss process in buildings, thus increasing our understanding of building thermal behaviour.

The approach presents an opportunity for designers to develop design solutions that are ideally matched to the heat loss field, thereby changing the culture in the industry from top-down to bottom-up solutions that are better than the simplified conventional solutions.

This article has illustrated the creation of an alternative bottom-up approach for recreating a complex natural phenomenon. It has also illustrated how the resulting self-organised emergent pattern 
can be utilized as a starting point for design exploration. It has shown that solutions based on the self-organised patterns generated in this research provide a higher thermal performance compared to conventionally designed solutions. In doing so, this article proposes the use of alternative approaches such as that investigated in this research, to develop solutions that are closer to nature, replacing the need to solve complex systems of equations.

Although this approach requires further developments, such as improving the object resolution and rendering speed, it has shown that it can act in a more direct and interactive way to generate solutions. Our future work will also investigate more complex building configurations and varying initial and boundary conditions.

Author Contributions: Conceptualization, P.B. and L.J.; methodology, P.B. and L.J.; formal analysis, L.J.; investigation, P.B. and L.J.; writing-original draft preparation, P.B.; writing-review and editing, L.J.; visualisation, P.B.; supervision, L.J.; All authors have read and agreed to the published version of the manuscript.

Funding: This research received no external funding. Generous support was received from the School of Creative Arts of the University of Hertfordshire in respect of the development of, and experimentation with, the model introduced in this article.

Conflicts of Interest: The authors declare no conflicts of interest.

\section{References}

1. Bendsøe, M.P. Optimal shape design as a material distribution problem. Struct. Optim. 1989, 1, $193-202$. [CrossRef]

2. Gersborg-Hansen, A.; Sigmund, O.; Haber, R.B. Topology optimization of channel flow problems. Struct. Multidiscip. Optim. 2005, 30, 181-192. [CrossRef]

3. Maute, K.; Allen, M. Conceptual design of aeroelastic structures by topology optimization. Struct. Multidiscip. Optim. 2004, 27, 27-42. [CrossRef]

4. Wolfram, S. A New Kind of Science; Wolfram Media: Champaign, IL, USA, 2002.

5. Jankovic, L. Changing the Culture of Building Simulation with Emergent Modelling. In Proceedings of the BS 2017: 15th Conference of the International Building Performance Simulation Association, San Francisco, CA, USA, 7-9 August 2017; pp. 222-229.

6. Malina, R.F.; Kauffman, S. At Home in the Universe: The Search for the Laws of Self-Organization and Complexity. Leonardo 1996, 29, 333. [CrossRef]

7. Bharadwaj, P.; Jankovic, L. Cellular Automata Simulations of Three-dimensional Building Heat Loss Field. In Proceedings of the BS 2019: 16th Conference of the International Building Performance Simulation Association, Rome, Italy, 2-4 September 2019.

8. Kauffman, S.A. Investigations; University Press: Oxford, NY, USA, 2000.

9. Hardy, J.; Pomeau, Y.; de Pazzis, O. Time Evolution of a Two-Dimensional Classical Lattice System. Phys. Rev. Lett. 1973, 31, 276-279. [CrossRef]

10. Frisch, U.; Hasslacher, B.; Pomeau, Y. Lattice-Gas Automata for the Navier-Stokes Equation. Phys. Rev. Lett. 1986, 56, 1505-1508. [CrossRef] [PubMed]

11. Salem, J.; Wolfram, S. Thermodynamics and Hydrodynamics of Cellular Automata. In Cellular Automata and Complexity; CRC Press: Boca Raton, FL, USA, 2018.

12. Wolfram, S. Cellular automaton fluids 1: Basic theory. J. Stat. Phys. 1986, 45, 471-526. [CrossRef]

13. Toffoli, T.; Margolus, N. Cellular Automata Machines: A New Environment for Modeling; MIT Press: Cambridge, MS, USA, 1987.

14. Frazer, J. An Evolutionary Architecture; Architectural Association: London, UK, 1995.

15. Jankovic, S.; Chan, A.H.C.; Jankovic, L.; Little, G.H. Bottom-Up Virtual Reality Model of a Shallow Two-Bar Truss with Snap-Through Behaviour. In Proceedings of the ACME 2000, London, UK, 16-19 April 2000.

16. Jankovic, L.; Jankovic, S.; Chan, A.H.C.; Little, G.H. Can bottom-up modelling in virtual reality replace conventional structural analysis methods? Autom. Constr. 2003, 12, 133-138. [CrossRef]

17. Jankovic, L. An Emergence-based Approach to Designing. Des. J. 2012, 15, 325-346. [CrossRef] 
18. Bharadwaj, P.; Jankovic, L.; Basurra, S. Developing A Bottom-up Approach for Delivering Innovative Zero Carbon Retrofit Solutions Using Agent-Based Modelling. In Proceedings of the Adaptive and interactive buildings and districts, Helsinki, Finland, 27-29 September 2017.

19. IES. Virtual Environment 2019. Available online: https://www.iesve.com/VE2019 (accessed on 18 March 2018).

20. Unity Technologies. Unity Game Development Platform. Available online: https://unity3d.com (accessed on 3 March 2020).

21. DesignBuilder Software Ltd. DesignBuilder. Available online: https://designbuilder.co.uk/ (accessed on 26 March 2019).

22. Kornicki, T. AnTherm-Heat \& Vapour Transfer-Thermal Bridges, Product Version 10.137.1.19821, 2003. Available online: http://antherm.eu/ (accessed on 29 June 2020).

23. Diamond, J.M. Guns, Germs and Steel-A Short History of Everybody in the Last 13,000 Years; Vintage: London, UK, 2005.

(C) 2020 by the authors. Licensee MDPI, Basel, Switzerland. This article is an open access article distributed under the terms and conditions of the Creative Commons Attribution (CC BY) license (http://creativecommons.org/licenses/by/4.0/). 